\title{
INVESTIGACIÓN
}

Recibido: 12/03/2021 --- Aceptado: 15/04/2021 --- Publicado: 24/05/2021

\section{PROPUESTAS PARA UNAS ESTRATEGIAS DE MARKETING EN REDES SOCIALES, MÁS EFICIENTES. EL ANÁLISIS DE LAS CUENTAS CORPORATIVAS UNIVERSITARIAS}

\section{Proposals for more efficient social media marketing strategies. The analysis of university corporate accounts}

(D) Luis Matosas-López: Universidad Rey Juan Carlos. España.

luis.matosas@urjc.es

(8)Elena Cuevas-Molano: Universidad Rey Juan Carlos. España. elena.cuevas@urjc.es

\section{Como citar el artículo:}

Matosas-López, L. y Cuevas-Molano, E. (2021). Propuestas para unas estrategias de marketing en redes sociales, más eficientes. El análisis de las cuentas corporativas universitarias.Vivat Academia. Revista de Comunicación, 154, 409-428. http://doi.org/10.15178/va.2021.154.e1358

\section{RESUMEN}

El presente trabajo examina los aspectos a considerar para alcanzar estrategias de marketing en redes sociales más eficientes. Los autores revelan las variables que llevan al usuario a reconocer, empleando retuits y favoritos, las publicaciones realizadas por las cuentas corporativas de instituciones universitarias. La investigación explora una muestra de diez universidades españolas y un total de 18.092 publicaciones, en la red social Twitter. El estudio adopta una metodología cuantitativa en la que se examinan treinta variables. Los investigadores llevan a cabo un análisis descriptivo y dos regresiones lineales; revelando: (a) las tendencias de uso habituales, y (b) las variables que inciden en el reconocimiento del contenido publicado a través de retuits, por un lado, y de favoritos, por otro. Los resultados corroboran la existencia de dos modelos de regresión robustos. El primero ( $\mathrm{p}$-valor < ,0001 y $\mathrm{R}^{2}=$,792) muestra cómo el reconocimiento de las publicaciones mediante retuits viene determinado por el uso de enlaces y hashtags. El segundo ( $\mathrm{p}$-valor <

\footnotetext{
${ }^{1}$ Luis Matosas-López: Doctor en Economía, Máster en Marketing, Licenciado en Investigación y Técnicas de Mercado y Diplomado en Ciencias Empresariales. Entre sus trabajos destacan artículos publicados en revistas internacionales indexadas en JCR y SJR así como capítulos de libro en editoriales como Tirant Lo Blanch, Pirámide, McGraw Hill, Dykinson, o Tecnos.
} 
Matosas-López, L. y Cuevas-Molano, E.

Propuestas para unas estrategias de marketing en redes sociales, más eficientes. El análisis de las cuentas corporativas universitarias

,0001 y $\mathrm{R}^{2}=$,886), por su parte, revela que el reconocimiento del contenido en forma de favoritos está condicionado por el volumen de publicaciones diarias y las publicaciones realizadas de 8:00 a 10:00 am. Los hallazgos de la presente investigación proporcionan, a académicos y profesionales, una visión actualizada de cuáles son las variables que inciden en estos indicadores de reconocimiento y que, por consiguiente, conducen a estrategias de marketing en redes sociales más eficientes.

PALABRAS CLAVE: Estrategia - Marketing - Medios sociales - Redes sociales Twitter - Gestión - Eficiencia - Regresiones lineales - Engagement.

\section{ABSTRACT}

The present work examines the aspects to take into consideration for more efficient marketing strategies in social network services. The authors address the variables that lead users to acknowledge, through retweets and favorites, the content published on the corporative accounts of university institutions. This research analyses 18.092 posts in a sample of ten Spanish universities, in Twitter. The study uses a quantitative methodology considering thirty variables. The researchers carry out a descriptive analysis and two linear regressions; revealing: (a) the usual trends of use, and (b) the variables that improve the acknowledgment of the content published through retweets, on the one hand, and favorites, on the other. The results corroborate the existence of two robust regression models. The first one ( $p$-value < ,0001 and $\mathrm{R}^{2}=$,792) shows how the acknowledgment through retweets is determined by the use of links and hashtags. Similarly, the second ( $\mathrm{p}$-value $<, 0001$ and $\mathrm{R}^{2}=, 886$ ) reveals that the acknowledgment of content in the form of favorites is conditioned by the daily volume of posts and the messages published from 8:00 to 10:00 am. The findings of this research provide, both academics and professionals, with an up-todate view of the variables that affect this acknowledgment indicators and that, consequently, lead to more efficient marketing strategies in social network services.

KEYWORDS: Strategy - Marketing- Social media - Social network services -Twitter - Management - Efficiency - Linear regressions - Engagement.

\section{INTRODUCCIÓN}

En las últimas décadas, la expansión de las tecnologías de la información y la comunicación (TIC) ha impulsado numerosos cambios sociales en todo el mundo. Entre estos cambios sobresale el de la integración de las redes sociales en el conjunto de la ciudadanía. En el caso particular de España, la generalización del empleo de redes sociales lleva, hoy, a dos de cada tres personas a acceder con regularidad a plataformas como Facebook o Twitter (Matosas-López y Romero-Ania, 2020).

El éxito de las redes sociales se debe a multitud de factores; pero entre ellos destacan aspectos como: el dinamismo del contenido, su utilidad colaborativa, su manejo intuitivo, su fácil acceso, o su carácter interactivo (Castaño et al., 2015;

Vivat Academia. Revista de Comunicación. 2021, n 154, 409-428 
Matosas-López, L. y Cuevas-Molano, E.

Propuestas para unas estrategias de marketing en redes sociales, más eficientes. El análisis de las cuentas corporativas universitarias

Gómez-García et al., 2020). Tal éxito ha llevado a la comunidad académica a realizar numerosos trabajos sobre esta cuestión. Así, por ejemplo, la revisión de la literatura realizada por Almansa et al. (2013), en torno a la investigación sobre redes sociales, diferencia tres grandes áreas temáticas: (a) la representación de los usuarios y la generación de vínculos entre estos (Junco, 2012; McAndrew y Jeong, 2012); (b) la estructuración de la red entorno a interés y motivaciones (Backstrom et al., 2006; Liu et al., 2006); y (c) la privacidad y los riesgos del medio (Calvete et al., 2010; McBride, 2011).

La dimensión alcanzada por este fenómeno ha transformado la manera de comunicarnos y de interactuar con nuestro entorno, hasta el punto de hacer necesaria la creación de un término descriptivo del usuario habitual en estas plataformas. Este es el término de "prosumidor mediático". El término "prosumidor", acuñado originariamente durante la década de los setenta, sirve para describir a aquel sujeto particularmente activo en contextos de producción y consumo en los que la tecnología tiene un papel relevante (McLuhan y Nevitt, 1972). De manera más reciente, y a partir de este concepto, otros autores han creado el término "prosumidor mediático" para hacer referencia al usuario acostumbrado al entorno de las redes sociales.

Sandoval Romero y Aguaded Gómez (2012), por ejemplo, describen al "prosumidor mediático" como aquel sujeto capaz de tomar el protagonismo del medio, produciendo y consumiendo información para generar una cultura participativa. Sánchez Carrero y Contreras Pulido (2012), por su parte, definen al "prosumidor mediático" como aquel usuario que asume de manera activa el rol del propio canal de comunicación, aprovechando el mismo para convertirse en generador de opiniones sobre una variedad de temas. En la misma línea, García-Ruíz et al. (2014) apuntan que el "prosumidor mediático" es un individuo activo y competente a la hora de analizar y elaborar contenidos críticos y creativos de manera autónoma.

Uno de los enfoques empleados por los investigadores para examinar el comportamiento y las respuestas del "prosumidor mediático" en estas plataformas es el de la Teoría de Uso y Gratificación. Esta teoría, desarrollada en su origen para describir cómo las audiencias interactúan con otros medios masivos como la radio, la prensa o a televisión (Katz et al., 1974; Ruggiero, 2000), se ha aplicado en los últimos años en multitud de trabajos sobre la utilización de redes sociales con distintos fines (Chen, 2011; Cuevas-Molano et al., 2019; García-Ruiz et al., 2018; Raacke y BondsRaacke, 2008; Smock et al., 2011).

El marco conceptual definido por la Teoría de Uso y Gratificación explora la forma en la que el individuo emplea los medios de comunicación masivos para satisfacer ciertas necesidades y motivaciones. De acuerdo con Rubin (1994), esta teoría se sustenta en cinco pilares: (1) la selección y uso del medio tiene una finalidad; (2) el sujeto es el que toma la iniciativa seleccionando el medio con el objetivo de obtener una gratificación; (3) el comportamiento está condicionado por diferentes factores 
Matosas-López, L. y Cuevas-Molano, E.

Propuestas para unas estrategias de marketing en redes sociales, más eficientes. El análisis de las cuentas corporativas universitarias

sociales; (4) existen distintas alternativas mediáticas que compiten entre sí en términos de selección, uso y gratificación; y (5) el individuo tiene una posición relevante dentro del medio.

La naturaleza de las redes sociales encaja a la perfección con los supuestos establecidos para la citada teoría. Los usuarios, teniendo otras posibilidades, eligen libremente estas plataformas; acceden a las mismas con el propósito de lograr una gratificación; se comunican condicionados por aspectos sociológicos y gozan de una posición de empoderamiento en la plataforma. Por consiguiente, el potencial de las redes sociales para propagar información a grandes audiencias, tal y como sucede con otros medios masivos (prensa, radio o televisión), hace que el enfoque de la Teoría de Uso y Gratificación sea particularmente adecuado para la contextualización de las investigaciones en este campo.

\subsection{Las redes sociales en el ámbito de las estrategias de marketing}

En el ámbito de las estrategias de marketing, en el que se contextualiza el presente trabajo, los usuarios recurren a estas plataformas con el objetivo de gratificar o satisfacer su necesidad de comunicación con la organización o marca representada por la cuenta (Matosas-López, 2020).

El potencial de estas tecnologías en el contexto de las estrategias de marketing resulta innegable; es por ello que las redes sociales han captado la atención de organizaciones de todo tipo. Desde que estas plataformas aparecieran en nuestras vidas a principios del año 2000, organizaciones de diversa índole han recurrido a ellas como instrumento para conectar con su audiencia o público objetivo.

En este sentido, organizaciones empresariales, por un lado, y universitarias, por otro, son dos de los entes en los que las redes sociales han experimentado un mayor desarrollo (Alonso García y Alonso García, 2014; Castelló Martínez, 2012; García García, 2018; Godoy Martín, 2012; Matosas-López et al., 2021). Tanto en unas, como en otras, plataformas como Twitter, Facebook o Instagram se han integrado en las distintas estrategias corporativas, hasta convertirse, en muchos casos, en piedra angular de las acciones llevadas a cabo en departamentos de marketing y comunicación.

La revisión de la literatura sobre la cuestión del uso de redes sociales en el ámbito de las estrategias de marketing, tanto en la empresa como en la universidad, deja un sin fin de referencias. En lo que atañe al uso de redes sociales en el contexto empresarial, los autores destacan los trabajos de: Alonso (2015), Matosas López (2018), Mukherjee y Banerjee (2019), Kraus et al. (2019), o Giakoumaki y Krepapa (2019).

El trabajo de Alonso (2015) examina la actividad de distintas marcas de moda dentro de las redes sociales Facebook, Twitter, Pinterest e Instagram. En este estudio, la autora explora las publicaciones realizadas, los mensajes viralizados y la tipología 
Matosas-López, L. y Cuevas-Molano, E.

Propuestas para unas estrategias de marketing en redes sociales, más eficientes. El análisis de las cuentas corporativas universitarias

de contenido empleado por las cuentas corporativas de cada marca, en las cuatro plataformas observadas.

Por su parte, el estudio de Matosas López (2018), analiza el tipo de variables que condicionan la eficiencia de las estrategias de marketing de empresas del sector alimenticio, en Twitter. En este trabajo, el autor examina la manera en que aspectos como la interactividad del contenido (enlaces o menciones), la vivacidad de las publicaciones (fotos o hashtags), la valencia del sentimiento de los emoticonos, o la hora de envío influyen en la difusión de las publicaciones.

La investigación de Mukherjee y Banerjee (2019), apoyándose en cuestionarios, analiza el impacto que las inserciones publicitarias en Facebook tienen en los usuarios de la plataforma. Los autores demuestran que la publicidad en esta red puede generar en la audiencia una actitud positiva hacia la marca, aumentando además la intención de compra de los productos o servicios de la compañía.

Kraus et al. (2019), por su parte, examinan, a través de entrevistas personales, los protocolos de creación de contenido seguidos por una muestra de PYMES (pequeñas y medianas empresas) en sus páginas de empresa en Facebook. Los autores concluyen que, a pesar de la limitación de recursos (tanto humanos como económicos), estas empresas son conscientes de los aspectos a considerar en la gestión de sus estrategias de marketing en este medio.

Por último, el estudio de Giakoumaki y Krepapa (2019) analiza como los contenidos de marcas de lujo en la plataforma Instagram pueden generar un mayor o menor engagement hacia la marca dependiendo de si la publicación proviene de una $u$ otra fuente. En este trabajo, los autores constatan que el compromiso alcanzado mejora cuando la fuente de la que proviene el mensaje es un usuario particular, y no un influencer o la propia cuenta corporativa de la marca.

Por otro lado, en lo que se refiere a los estudios que toman como objeto de observación organizaciones universitarias, los autores destacan los trabajos de: Laaser et al. (2012), Valerio Ureña et al. (2014), Puertas Hidalgo y Carpio Jiménez (2016), Kimmons et al. (2017), o Wu et al. (2019).

El trabajo de Laaser et al. (2012) analiza, mediante entrevistas a expertos en gestión universitaria, el uso de las redes sociales Facebook, Twitter, LinkedIn y Google+ como herramienta de marketing. En este estudio, los autores revelan la existencia de problemas en los equipos de gestión y falta de visión estratégica en el uso de estas tecnologías, además de la necesidad de aplicar indicadores de eficiencia y alcance.

La investigación de Valerio Ureña et al. (2014) examina, en Facebook, el engagement generado entre una muestra de organizaciones universitarias y sus respectivas audiencias, en función del momento de publicación de los mensajes. Si bien los resultados muestran que el grueso de la actividad se centra en los días y horarios laborales, los investigadores apuntan también que muchos de los contenidos con

Vivat Academia. Revista de Comunicación. 2021, n 154, 409-428 
Matosas-López, L. y Cuevas-Molano, E.

Propuestas para unas estrategias de marketing en redes sociales, más eficientes. El análisis de las cuentas corporativas universitarias

mejor aceptación son aquellos que los Community managers programan fuera de esos periodos.

Puertas Hidalgo y Carpio Jiménez (2016) analizan el uso que las universidades hacen de las plataformas Facebook, Twitter e Instagram en el marco de sus estrategias corporativas. Los autores apuntan que estas plataformas y el engagement creado a partir de ellas pueden actuar como aliados a la hora alcanzar los objetivos de la organización.

La investigación de Kimmons et al. (2017), tras examinar las métricas de actividad de una muestra de instituciones universitarias en Twitter, apunta que aun cuando las redes sociales sirven reforzar las estrategias de marketing de estas organizaciones, su alcance real es limitado. Los autores revelan que la mayor parte de los mensajes de estas instituciones son unidireccionales, carentes de sentimiento y centrados en una reducida variedad de temas.

Por último, la investigación de Wu et al. (2019) estudia el dialogo entre universidad y público objetivo a través del análisis de las publicaciones y comentarios de ambos en la plataforma Facebook. Los autores demuestran que las publicaciones que emplean un tono amigable fomentan el acercamiento entre organización y usuario, recibiendo más comentarios que aquellas que recurren a un tono directo y autoritario.

\section{OBJETIVOS}

A pesar de que son muchos los trabajos sobre estrategias de marketing en redes sociales, las claves para mejorar la eficiencia de estas estrategias siguen sujetas a debate. Si bien es cierto que académicos y profesionales coinciden en que el indicador que sirve para baremar la eficiencia de las acciones de marketing en este medio es el reconocimiento que la audiencia otorga a la organización, al compartir o marcar como favorito el contenido publicado por esta (Chen, 2011; Tafesse, 2015); la manera de mejorar este indicador continua siendo objeto de estudio.

El trabajo que nos ocupa pretende arrojar luz sobre esta cuestión; proporcionando una visión actualizada de cuáles son las variables que inciden en este indicador y que, por consiguiente, permiten a cualquier organización llevar a cabo estrategias de marketing en redes sociales más eficientes.

La presente investigación toma como objeto de exploración las cuentas corporativas de las organizaciones universitarias españolas en la plataforma Twitter. En este contexto, los investigadores analizan, por un lado, cuáles son las tendencias de uso más habituales y, por otro, cuáles son las variables que inciden en el reconocimiento del contenido publicado (ya sea compartiendo la publicación o marcándola como favorita). Por consiguiente, los autores plantean dos preguntas de investigación diferenciadas. Estos interrogantes, considerando las particularidades de la plataforma observada, quedan formulados de la siguiente manera: 
Matosas-López, L. y Cuevas-Molano, E.

Propuestas para unas estrategias de marketing en redes sociales, más eficientes. El análisis de las cuentas corporativas universitarias

PI1: ¿Cuáles son los volúmenes, componentes y momentos de publicación más habituales?

PI2: ¿Cuáles son los volúmenes, componentes y momentos de publicación que inciden en el reconocimiento del contenido publicado en términos de retuits y favoritos?

Estas dos preguntas nos llevan, en última instancia, a realizar las dos siguientes reflexiones sobre las estrategias de marketing en redes sociales. Primero ¿qué se hace habitualmente? y segundo ¿qué se debería hacer? Estas dos reflexiones son, en realidad, las que subyacen detrás de los interrogantes de investigación planteados, y las que serán abordadas en la parte final del trabajo.

\section{METODOLOGÍA}

\subsection{Muestra}

Los investigadores escogen los elementos muestrales de entre las ochenta y dos instituciones universitarias oficialmente reconocidas por el Ministerio de Educación y Formación Profesional (MEFP) en España. La selección de elementos muestrales se realiza tomando como referencia dos rankings ampliamente reconocidos por la comunidad académica a la hora de examinar la actividad del estamento universitario. Por un lado, el listado Webometrics, ranking desarrollado por el Laboratorio de Cibermetría del Consejo Superior de Investigaciones Científicas en España (CSIC) y, por otro, el Academic Ranking of World Universities (ARWU) o ranking de Shanghái (Marciniak, 2013; Túñez López et al., 2015).

Los investigadores parten de las universidades emplazadas en las quince primeras posiciones del listado Webometrics en España durante el año 2019, para a continuación cotejar si estas organizaciones aparecen a su vez entre las 500 primeras del ranking ARWU de ese mismo año. Los autores, tras esta comprobación, se quedan solo con aquellas universidades que, además de estar entre las quince primeras de España en el listado Webometrics, aparecen también entre las 500 primeras del mundo de acuerdo con el baremo del ranking ARWU.

Esto reduce los elementos muestrales a un total de diez. Las instituciones seleccionadas para formar la muestra fueron: Universidad de Barcelona, Universidad Complutense de Madrid, Universidad Autónoma de Barcelona, Universidad de Valencia, Universidad de Granada, Universidad Autónoma de Madrid, Universidad Politécnica de Catalunya, Universidad Politécnica de Valencia, Universidad Politécnica de Madrid y Universidad Pompeu Fabra.

\subsection{Extracción de datos}

Seleccionada la muestra de diez universidades, los investigadores extraen de la plataforma Twitter todo el contenido publicado por las cuentas corporativas oficiales

Vivat Academia. Revista de Comunicación. 2021, n 154, 409-428 
Matosas-López, L. y Cuevas-Molano, E.

Propuestas para unas estrategias de marketing en redes sociales, más eficientes. El análisis de las cuentas corporativas universitarias

de las diez instituciones, en un período de un año (del 31 de diciembre de 2019 al 31 de diciembre de 2020). Dado que se analizan de manera general las estrategias de marketing y comunicación de la universidad, contemplando todas sus vertientes y el conjunto de su actividad - investigación, transferencia del conocimiento, acción social hacia sus stakeholders (administraciones públicas, empresas, etc.) - y esta actividad, a diferencia de la docente, no se concentran en un periodo concreto del año, los autores toman como rango de estudio el año natural y no el académico.

La extracción de datos se realiza utilizando el proveedor de servicios Twitonomy (Guijarro et al., 2018; Quintana Pujalte et al., 2018); recopilando un total de 18.092 publicaciones (tuits originales, retuits y respuestas), además del reconocimiento alcanzado por cada publicación en términos de retuits y favoritos.

El conjunto de datos recopilado, a través del proveedor de servicios, se exporta y almacena en una base de datos para su posterior análisis.

\subsection{Análisis de datos}

Los autores abordan el análisis de los datos extraídos adoptando un enfoque cuantitativo, en el que consideran un total de treinta variables (ver Figura 1). En la investigación, se llevan a cabo dos tipos de análisis. Por un lado, se realiza un análisis descriptivo y, por otro, un análisis de regresión lineal múltiple.

La exploración descriptiva, en línea con Wu et al. (2019), pretende contestar la primera pregunta de investigación (PI1). Aquí, los autores desarrollan su análisis sobre las veintiocho variables catalogadas en la Figura 1 como variables independientes. Estas veintiocho variables se agrupan en cuatro categorías: volúmenes de publicación, componentes de la publicación, publicaciones por día de la semana y publicaciones por franja horaria.

El análisis de regresión lineal múltiple, en línea con Matosas-López y RomeroAnia (2020), persigue dar respuesta a la segunda pregunta de investigación (PI2). En este análisis, los autores toman las veintiocho variables independientes y las dos dependientes indicadas en la Figura 1. Las variables independientes son las agrupadas en las categorías antes mencionadas, mientras que las variables independientes son: las publicaciones retuiteadas y las publicaciones marcadas como favoritas. 
Matosas-López, L. y Cuevas-Molano, E.

Propuestas para unas estrategias de marketing en redes sociales, más eficientes. El análisis de las cuentas corporativas universitarias

VARIABLES

INDEPENDIENTES

VARIABLES

DEPENDIENTES

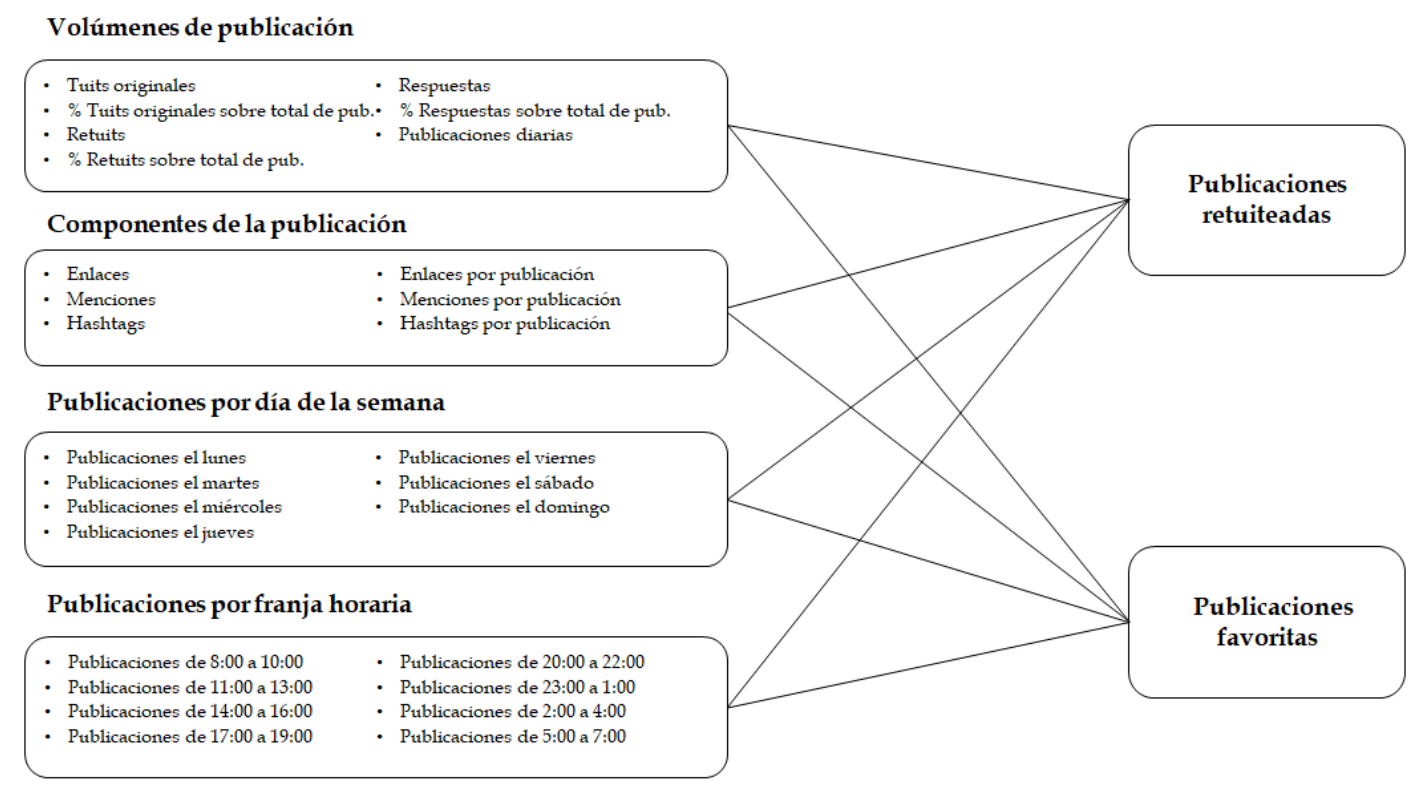

Figura 1. Variables consideradas y modelo objeto de estudio

Fuente: Elaboración propia

Todos los análisis mencionados anteriormente se llevaron a cabo empleando el software de examen estadístico IMB SPSS en su versión 27.

\section{RESULTADOS}

\subsection{Volúmenes, componentes y momentos de publicación más habituales (PI1)}

En el análisis descriptivo llevado a cabo para dar respuesta a la primera pregunta de investigación destacan los siguientes datos. En lo que atañe a los volúmenes de publicación, cabe mencionar que, si bien el 30,28\% de las publicaciones son retuits aprovechando el contenido de otras cuentas, la mayoría de las publicaciones son contenidos originales diseñados por la propia universidad (55,75\%). En esta categoría destaca también que el promedio de mensajes diarios es de 5,901. No obstante, cabe señalar que en estas variables se observa una elevada dispersión de datos, lo cual denota la existencia de patrones de actuación distantes entre universidades.

En lo que se refiere a los componentes de las publicaciones, las menciones y los hashtags son los elementos que aparecen de forma más recurrente. En estos ítems se observan promedios por mensaje de, 701 y, 899 respectivamente.

Por último, en cuanto al momento de publicación, las frecuencias más elevadas se detectan en la parte central de la semana (martes, miércoles y jueves) y durante las franjas horarias de la mañana (de 8:00 a 13:00). 
Matosas-López, L. y Cuevas-Molano, E.

Propuestas para unas estrategias de marketing en redes sociales, más eficientes. El análisis de las cuentas corporativas universitarias

Tabla 1. Resultados descriptivos de volúmenes, componentes y momentos de publicación

\begin{tabular}{|c|c|c|c|}
\hline Categoría / Variable & Promedio & Desviación típica & $\%$ sobre total de pub. \\
\hline \multicolumn{4}{|l|}{ Volúmenes de publicación } \\
\hline Tuits originales & 891,210 & 584,600 & - \\
\hline$\%$ Tuits originales sobre total de pub. & - & - & 55,75 \\
\hline Retuits & 427,654 & 485,214 & - \\
\hline$\%$ Retuits sobre total de pub. & - & - & 30,28 \\
\hline Respuestas & 170,231 & 402,521 & - \\
\hline$\%$ Respuestas sobre total de pub. & - & - & 13,97 \\
\hline Publicaciones diarias & 5,901 & 3,257 & - \\
\hline \multicolumn{4}{|l|}{ Componentes de la publicación } \\
\hline Enlaces & 602,001 & 547,190 & - \\
\hline Menciones & 1125,158 & 1058,241 & - \\
\hline Hashtags & 1502,374 & 1777,830 & - \\
\hline Enlaces por publicación &, 582 & ,182 & - \\
\hline Menciones por publicación & ,701 &, 530 & - \\
\hline Hashtags por publicación & 899 & ,601 & - \\
\hline \multicolumn{4}{|l|}{ Publicaciones por día de la semana } \\
\hline Publicaciones en lunes & 286,512 & 94,190 & - \\
\hline Publicaciones en martes & 374,214 & 107,830 & - \\
\hline Publicaciones en miércoles & 320,321 & 100,280 & - \\
\hline Publicaciones en jueves & 340,320 & 99,570 & - \\
\hline Publicaciones en viernes & 287,321 & 100,140 & - \\
\hline Publicaciones en sábado & 52,652 & 28,352 & - \\
\hline Publicaciones en domingo & 32,740 & 14,280 & - \\
\hline \multicolumn{4}{|l|}{ Publicaciones por franja horaria } \\
\hline Publicaciones de 8:00 a 10:00 & 429,4 & 200,47 & - \\
\hline Publicaciones de 11:00 a 13:00 & 401,4 & 195,32 & - \\
\hline Publicaciones de 14:00 a 16:00 & 274 & 157,16 & - \\
\hline Publicaciones de 17:00 a 19:00 & 140,4 & 84,40 & - \\
\hline Publicaciones de 20:00 a 22:00 & 28 & 34,46 & - \\
\hline Publicaciones de 23:00 a 1:00 & 1,2 & 1,74 & - \\
\hline Publicaciones de 2:00 a 4:00 & ,90 & 1,01 & - \\
\hline Publicaciones de 5:00 a 7:00 & 120,7 & 90,97 & - \\
\hline
\end{tabular}

Fuente: Elaboración propia.

\subsection{Volúmenes, componentes y momentos de publicación que inciden en el reconocimiento del contenido publicado en términos de retuits y favoritos (PI2)}

Para dar respuesta a la segunda pregunta de investigación se realizan dos análisis de regresión lineal múltiple. Uno para la variable dependiente publicaciones retuiteadas y otro para la variable dependiente publicaciones favoritas (ver Figura 1). Los resultados obtenidos, a partir de estos dos análisis de regresión, permiten identificar las variables que indicen en el reconocimiento del contenido, en forma de 
Matosas-López, L. y Cuevas-Molano, E.

Propuestas para unas estrategias de marketing en redes sociales, más eficientes. El análisis de las cuentas corporativas universitarias

retuits y favoritos. Con el propósito de examinar la influencia de cada variable independiente, los ítems se agregan durante el análisis de forma individual, recurriendo al método de incorporación de variables en etapas sucesivas.

En la primera regresión, la realizada para la variable publicaciones retuiteadas, el ítem "Enlaces" $(\beta=, 599$, $p$-valor $<, 0001)$ ingresa en el primer paso del procedimiento. La variable "Hashtags" $(\beta=, 501$, p-valor $<, 005)$, por su parte, se agrega en el segundo paso. El modelo para esta primera variable dependiente resulta significativo en su conjunto $(\mathrm{F}=69,548$, p-valor $<, 0001)$, explicando la varianza de la variable dependiente de manera óptima con un valor $\mathrm{R}^{2}=$,792. Por consiguiente, este primer análisis de regresión muestra el impacto de las variables "Enlaces" y "Hashtags" en el reconocimiento obtenido por el contenido publicado a través de retuits. (Ver Tabla 2).

En la segunda regresión, la desarrollada para la variable publicaciones favoritas, el ítem "Publicaciones diarias" $(\beta=1,000, p$-valor $<, 0001)$ aparece en el primer paso del proceso. La variable agregada, con signo negativo, en el segundo paso es la denominada "Retuits" ( $\beta=-, 601, \mathrm{p}$-valor $<, 0001)$. Por último, en tercer lugar, se incorpora el ítem "Publicaciones de 8:00 a 10:00" $(\beta=, 297$, p-valor $<, 005)$. En consecuencia, el modelo para la variable publicaciones favoritas resulta también significativo $(\mathrm{F}=299,871$, $\mathrm{p}$-valor $<, 0001)$, explicando adecuadamente la varianza de esta variable con un $\mathrm{R}^{2}=, 886$. Por ende, este segundo análisis de regresión revela la influencia de las variables "Publicaciones diarias", "Retuits" y "Publicaciones de 8:00 a 10:00" en el reconocimiento alcanzado, a través de favoritos. La primera y la tercera con signo positivo y la segunda, la que alude al uso de la funcionalidad de retuit, con valencia negativa (ver Tabla 2).

La Tabla 2 presenta, tan solo, los datos de aquellas variables que tras el análisis de regresión han demostrado tener una incidencia significativa sobre las variables independientes examinadas (Publicaciones retuiteadas y Publicaciones favoritas). El resto de las variables han sido eliminadas de la tabla con el propósito de facilitar la visualización de los resultados.

Tabla 2. Coeficientes significativos identificados en los análisis de regresión lineal múltiple

Publicaciones retuiteadas

Publicaciones favoritas

\begin{tabular}{lcccc}
\hline Categoría / Variable & Coeficiente $\boldsymbol{\beta}$ & p-valor & Coeficiente $\boldsymbol{\beta}$ & p-valor \\
\hline Volúmenes de publicación & & & &, $000^{* *}$ \\
\hline Retuits & - & - &,- 601 &, $000^{* *}$ \\
Publicaciones diarias & - & - & 1,000 & - \\
\hline Componentes de la publicación & & & & - \\
\hline Enlaces &, 599 &, $000^{* *}$ & - & - \\
Hashtags &, 501 &, $003^{*}$ & & -
\end{tabular}

Publicaciones por día de la semana

No se identificaron variables sigs. 
Matosas-López, L. y Cuevas-Molano, E.

Propuestas para unas estrategias de marketing en redes sociales, más eficientes. El análisis de las cuentas corporativas universitarias

\begin{tabular}{lllll}
\hline Publicaciones por franja horaria & & & \\
\hline Publicaciones de 8:00 a 10:00 & - & - &, 297 &, $003^{*}$ \\
\hline${ }^{*}$ p-valor $<, 005 /{ }^{* *}$ p-valor $<, 0001$. & &
\end{tabular}

Fuente: Elaboración propia.

En la presente tabla, el coeficiente $\beta$ indica el número de unidades que aumentarán o disminuirán - dependiendo del signo - las variables dependientes (Publicaciones retuiteadas y Publicaciones favoritas), por cada unidad que varíen las respectivas variables independientes. Definiendo, por consiguiente, el sentido de la relación entre unas y otras.

No obstante, el indicador, quizá, más relevante de la tabla que nos ocupa es el denominado p-valor. Este valor revela el nivel de significatividad de las relaciones entre variables, antes aludidas. Así, por ejemplo, serán más significativas aquellas relaciones en las que el p-valor sea menor de ,0001 y ligeramente más débiles, aunque también significativas, aquellas en las que el p-valor esté por debajo de ,005.

\section{DISCUSION}

Con anterioridad, diferentes autores han destacado la necesidad de que las organizaciones universitarias lleven a cabo estrategias de marketing en redes sociales más eficientes. Casanoves Boix et al. (2018), por ejemplo, apuntan que las universidades deben apoyarse en estas plataformas con el objeto potenciar el desarrollo de estrategias de construcción de marca. Estos investigadores señalan también que las cuentas corporativas universitarias requieren de una gestión totalmente profesionalizada.

En la misma línea, Guzmán Duque et al. (2012) señalan que las redes sociales deben ayudar a las universidades a mejorar su identidad corporativa y a consolidar su presencia en el territorio en el que están enraizadas. Estos autores apuntan además que estas tecnologías deben servir de apoyo en el diseño de campañas promocionales e incluso como herramienta de captación de estudiantes.

Los hallazgos del trabajo que nos ocupa proporcionan, a académicos y profesionales, el conocimiento necesario para llevar a cabo una gestión más eficiente de estas herramientas, dentro del ámbito universitario. Los resultados obtenidos por los autores permiten dar respuesta a las dos preguntas de investigación planteadas.

\subsection{Volúmenes, componentes y momentos de publicación más habituales (PI1)}

En lo que se refiere a la primera pregunta de investigación, sobresalen varios aspectos: (a) la preponderancia de las publicaciones originales (sobre aquellas que son retuits o respuestas), (b) un alto promedio de publicaciones diarias, (c) el uso intensivo de menciones y hashtags (incluso por encima del uso de enlaces) y (d) la 
Matosas-López, L. y Cuevas-Molano, E.

Propuestas para unas estrategias de marketing en redes sociales, más eficientes. El análisis de las cuentas corporativas universitarias

explotación de la parte central de la semana y el horario matinal como principal momento de publicación.

De entre estos hallazgos, los autores destacan la elevada presencia de menciones y hashtags en los mensajes. Estos resultados, están en concordancia con las investigaciones de autores como Puertas Hidalgo y Carpio Jiménez (2016) o Laaser et al. (2012). Estudios ambos en los que se enfatiza la importancia del uso de menciones, en el primero, y de hashtags, en el segundo.

No obstante, estos resultados difieren, por ejemplo, con lo apuntado por Túñez López et al. (2015) en su trabajo sobre el uso de Facebook y Twitter como canal de comunicación corporativa en el ámbito universitario. Investigación está en la que los autores destacan la importancia del enlace, por encima de la mención o el hashtag, en cualquier publicación. Este hecho, en opinión de los autores, podría apuntar hacia una evolución de las estrategias de marketing y comunicación en redes sociales, revelando que el uso de menciones o hashtags, en la actualidad, gozaría de mayor aceptación que la utilización de enlaces.

Sea como fuere, resulta indiscutible que tanto menciones como hashtags otorgan un importante valor añadido a las publicaciones. En el caso de las menciones, el potencial reside en la implicación de la audiencia en un proceso de dialogo; mientras que en el caso de los hashtags, el interés radica en su poder para monitorizar el contenido dentro de la plataforma, haciéndolo más accesible para el público objetivo de la organización.

\subsection{Volúmenes, componentes y momentos de publicación que indicen en el reconocimiento del contenido publicado en términos de retuits y favoritos (PI2)}

En lo que al segundo interrogante de investigación atañe, cabe destacar que el reconocimiento alcanzado por los mensajes está influenciado por: (a) el uso de enlaces y hashtags en las publicaciones (cuando se trata del reconocimiento del contenido mediante retuits); y (b) el número de publicaciones diarias, los mensajes entre las 8:00 y las 10:00 am y los retuits realizados desde la cuenta (si hablamos del reconocimiento mediante favoritos).

La incidencia de las variables enlaces, hashtags, publicaciones diarias y mensajes entre las 8:00 y las 10:00 am tiene valencia positiva, es decir, un mayor uso de estos elementos influye positivamente en el reconocimiento del contenido mediante retuits y/o favoritos. Estos hallazgos están en la línea de otros estudios previos sobre estrategias de marketing en redes sociales, dentro del ámbito universitario (Laaser et al., 2012; Túñez López et al., 2015; Valerio Ureña et al., 2014). Estudios que, tras analizar la actividad en plataformas como Twitter y Facebook, entre otras, subrayan que aspectos como los anteriormente señalados condicionan el impacto y reconocimiento que el contenido publicado por la institución alcanza en su público objetivo. 
Matosas-López, L. y Cuevas-Molano, E.

Propuestas para unas estrategias de marketing en redes sociales, más eficientes. El análisis de las cuentas corporativas universitarias

La última variable que parece influir en el reconocimiento alcanzado por el contenido, en este caso de manera negativa, es el número de publicaciones retuiteadas por la universidad. Esta variable aparece en la regresión desarrollada para publicaciones favoritas con valencia negativa, indicando, por tanto, que, a mayor volumen de contenido retuiteado, menor reconocimiento en forma de favoritos. Este hecho está en consonancia con los hallazgos de Matosas López (2018) en su estudio sobre viralización de contenido en la red social Twitter. En este trabajo, el autor apunta que el excesivo uso de retuits en una cuenta corporativa puede ser percibido por la audiencia de esta como indicador de falta de originalidad e incluso debilidad. Hecho este que llevaría a sus seguidores a penalizar a la organización.

\section{CONCLUSIONES}

Los hallazgos de la presente investigación proporcionan datos que invitan a la reflexión. Si bien los resultados obtenidos como respuesta a las dos preguntas de investigación planteadas están, en líneas generales, alineados; también se observan evidencias contradictorias. Estos hallazgos contrapuestos reflejan la existencia de un desajuste entre lo que habitualmente hacen las cuentas corporativas (PI1) y lo que realmente deberían hacer para mejorar el reconocimiento alcanzado por sus publicaciones (PI2).

Ejemplo de la alineación en los hallazgos entre interrogantes de investigación son las variables hashtags y publicaciones entre las 8:00 y las 10:00 am. Por un parte, los datos de la PI1 muestran la utilización recurrente de ambos elementos. Por otra, los hallazgos de la PI2 revelan que ambas variables favorecen el reconocimiento del contenido. Nos encontraríamos aquí en un escenario óptimo de gestión de las estrategias de marketing en redes sociales. Es decir, las variables que habitualmente se emplean coinciden con aquellas que han demostrado tener una incidencia significativa en el reconocimiento de las publicaciones. Por tanto, lo que habitualmente se hace, coindice con lo que se debe hacer.

En el polo opuesto, ejemplo de resultados contrapuestos y de desajuste entre lo que se hace y lo que se debería hacer, son los datos relativos al uso de enlaces y retuits. Por una parte, los resultados para la PI1 revelan que el uso de enlaces es residual y que la utilización de retuits es recurrente. Por otra, los hallazgos de la PI2 indican que mientras la utilización de enlaces tiene un impacto positivo, el uso de retuits tiene una influencia negativa en el reconocimiento de las publicaciones. Estamos, por tanto, ante un escenario en el que, por un lado, se minimiza la utilización de un elemento (enlaces) que tiene una incidencia positiva en el reconocimiento y, por otro, se enfatiza el uso de una variable (retuits) que tiene un impacto negativo en ese reconocimiento. Estaríamos, por tanto, ante un escenario de gestión deficiente en las estrategias de marketing en redes sociales. Lo que habitualmente se hace no concuerda con lo que se debería hacer.

\subsection{Propuestas para unas estrategias de marketing en redes sociales, más eficientes}


Matosas-López, L. y Cuevas-Molano, E.

Propuestas para unas estrategias de marketing en redes sociales, más eficientes. El análisis de las cuentas corporativas universitarias

Considerando lo anteriormente expuesto, los autores concluyen que, efectivamente existe un cierto desajuste entre la actividad habitualmente desarrollada por las cuentas universitarias y lo que deberían llevar a cabo para conseguir un mejor reconocimiento en sus publicaciones. Por consiguiente, a la vista de esta situación, los autores postulan las siguientes propuestas, cómo manera de mejorar la eficiencia de las estrategias en este medio.

1. Mantener una actividad homogénea y constante en la plataforma, empleando entre 5 y 6 publicaciones diarias.

2. Emplear, de manera preferente, tuits originales (los producidos por la propia universidad). Sin descuidar, por su puesto, las respuestas a las interpelaciones de otros usuarios. Hecho este último que contribuirá a establecer un dialogo entre las partes, potenciando el engagement entre organización y audiencia.

3. Reducir el número de retuits a contenidos generados por terceros. Técnica esta que puede resultar contraproducente, llegando a perjudicar la percepción que el usuario tiene de la cuenta.

4. Impulsar la utilización de hashtags, para facilitar la monitorización de contenido, y de enlaces. Este último elemento, empleado cómo generador de tráfico hacia el sitio corporativo, contribuirá, como técnica de SEO (Search Engine Optimization), a mejorar el posicionamiento web de la institución.

5. Mantener una actividad de publicación uniforme durante la semana. Al menos en lo que a días laborables atañe, minorando la intensidad durante el fin de semana.

6. Reforzar las publicaciones durante las primeras franjas horarias de la mañana, para mejorar la viralización de los mensajes.

El presente estudio, al margen de las mencionadas propuestas, viene a constatar la necesidad de profundizar en el conocimiento de los aspectos que inciden en la eficiencia de las estrategias de marketing en redes sociales. Corroborando la importancia que, para académicos y profesionales, tiene el hecho de contar con una visión actualizada de cuáles son las variables que inciden en la eficiencia de las acciones en este medio.

\section{REFERENCIAS}

Almansa, A., Fonseca, O., y Castillo, A. (2013). Redes sociales y jóvenes. Uso de Facebook en la juventud colombiana y española. Comunicar, 20(40), 127-135. https://doi.org/10.3916/C40-2013-03-03

Alonso García, S., y Alonso García, M. del M. (2014). Las redes sociales en las universidades españolas. Vivat Academia, 0(126), 54. https://doi.org/10.15178/va.2014.126.54-62 
Matosas-López, L. y Cuevas-Molano, E.

Propuestas para unas estrategias de marketing en redes sociales, más eficientes. El análisis de las cuentas corporativas universitarias

Alonso, M. (2015). Las redes sociales como canal de comunicación de las marcas de moda españolas. El caso de Zara, Mango y el Corte Inglés. Index Comunicación, 5(1), 77-105. https://t.ly/3KRx

Backstrom, L., Huttenlocher, D., Kleinberg, J., y Lan, X. (2006). Group formation in large social networks: Membership, growth, and evolution. Proceedings of 12th International Conference on Knowledge Discovery in Data Mining, 44-54. https://www.cs.cornell.edu/ lars/kdd06-comm.pdf

Calvete, E., Orue, I., Estévez, A., Villardón, L., y Padilla, P. (2010). Cyberbullying in adolescents: Modalities and aggressors' profile. Computers in Human Behavior, 26(5), 1128-1135. https://doi.org/10.1016/J.CHB.2010.03.017

Casanoves Boix, J., Küster Boluda, I., y Vila López, N. (2018). ¿Por qué las instituciones de educación superior deben apostar por la marca? Revista de Investigación Educativa, 37(1), 111-127. https://doi.org/10.6018/rie.37.1.291191

Castaño, C., Maiz, I., y Garay, U. (2015). Redes sociales y aprendizaje cooperativo en un MOOC. Revista Complutense de Educación, 26(Especial), 119-139. https://doi.org/10.5209/rev_RCED.2015.v26.46328

Castelló Martínez, A. (2012). Los portales verticales de marketing y publicidad en los $\begin{array}{llll}\text { medios } & \text { sociales. } & \text { Vivat } & \end{array}$ https://doi.org/10.15178/va.2011.117e.970-1001

Chen, G. M. (2011). Tweet this: A uses and gratifications perspective on how active Twitter use gratifies a need to connect with others. Computers in Human Behavior, 27(2), 755-762. https:// doi.org/10.1016/J.CHB.2010.10.023

Cuevas-Molano, E., Sánchez Cid, M., y Matosas-López, L. (2019). Análisis bibliométrico de estudios sobre la estrategia de contenidos de marca en los medios sociales. Comunicación y Sociedad, 2019, 1-25. https://doi.org/10.32870/cys.v2019i0.7441

García-Ruíz, R., Ramirez-Garcia, A., y Rodriguez-Rosell, M. (2014). Educación en alfabetización mediática para una nueva ciudadanía prosumidora. Comunicar, XXII(43), 15-23. https:// doi.org/10.3916/C43-2014-01

García-Ruiz, R., Tirado, R., y Hernando, Á. (2018). Redes sociales y estudiantes $\square$ : motivos de uso y gratificaciones . Evidencias para el aprendizaje. Aula Abierta, 47(3), 291-298. https:// doi.org/10.17811/rifie.47.3.2018.291-298

García García, M. (2018). Universidad y medios sociales. Gestión de la comunicación en la universidad española. Prisma Social, 22(3), 21-36. https://revistaprismasocial.es/article/view/2535 
Matosas-López, L. y Cuevas-Molano, E.

Propuestas para unas estrategias de marketing en redes sociales, más eficientes. El análisis de las cuentas corporativas universitarias

Giakoumaki, C., y Krepapa, A. (2019). Brand engagement in self-concept and consumer engagement in social media: The role of the source. Psychology and Marketing, 37(3), 457-465. https://doi.org/10.1002/mar.21312

Godoy Martín, F. J. (2012). Uso de los medios sociales en las empresas de $\begin{array}{llll}\text { comunicación andaluzas. Vivat Academia, } & 0(117 \mathrm{E}), & \end{array}$ https://doi.org/10.15178/va.2011.117e.1129-1142

Gómez-García, M., Matosas-López, L., y Palmero-Ruiz, J. (2020). Social Networks Use Patterns among University Youth: The Validity and Reliability of an Updated $\begin{array}{llll}\text { Measurement Instrument. } & \text { Sustainability, }\end{array}$ https://doi.org/10.3390/su12093503

Guijarro, E., Santandreu Mascarell, C. Canós Darós, L., Díez Somavilla, R., y Babiloni Griñón, E. (2018). Multicriteria techniques applied to social media in public sector: the use of Twitter by different law enforcement authorities profiles. Revista Latina de Comunicación Social, 73, 1412-1427. https://doi.org/10.4185/RLCS-2018-1314-73

Guzmán Duque, A. P., Del Moral Pérez, M. E., y González Ladron de Guevara, F. (2012). Usos de Twitter en las universidades iberoamericanas. Revista Latinoamericana de Tecnología Educativa - RELATEC, 11(1), 27-39. http://mascvuex.unex.es/revistas/index.php/relatec/article/view/845

Junco, R. (2012). The relationship between frequency of Facebook use, participation in Facebook activities, and student engagement. Computers y Education, 58(1), 162171. https:// doi.org/10.1016/j.compedu.2011.08.004

Katz, E., Blumler, J. G., y Gurevitch, M. (1974). Uses and Gratifications Research. The Public Opinion Quarterly, 37(4), 509-523. https:/ / doi.org/10.2307/2747854

Kimmons, R., Veletsianos, G., y Woodward, S. (2017). Institutional Uses of Twitter in U.S. Higher Education. Innovative Higher Education, 42(2), 97-111. https://doi.org/10.1007/s10755-016-9375-6

Kraus, S., Gast, J., Schleich, M., Jones, P., y Ritter, M. (2019). Content is King: How SMEs Create Content for Social Media Marketing Under Limited Resources. Journal of Macromarketing, 39(4), 415-430. https://doi.org/10.1177/0276146719882746

Laaser, W., Brito, J. G., y Toloza, E. A. (2012). El uso de redes sociales por parte de las universidades a nivel institucional. Un estudio comparativo. RED Revista de Educación a Distancia, 32(3), 231-239. https://www.um.es/ead/red/32/

Liu, H., Maes, P., y Davenport, G. (2006). Unraveling the Taste Fabric of Social Networks. International Journal on Semantic Web and Information Systems, 2(1), $42-71$. https://doi.org/10.4018/jswis.2006010102 
Matosas-López, L. y Cuevas-Molano, E.

Propuestas para unas estrategias de marketing en redes sociales, más eficientes. El análisis de las cuentas corporativas universitarias

Marciniak, R. (2013). Propuesta metodológica para la aplicación del benchmarking internacional en la evaluación de la calidad de la educación superior virtual. Revista de Universidad y Sociedad Del Conocimiento, 12(3), 46-61. https://doi.org/10.7238/rusc.v12i3.2163

Matosas-López, L. (2020). Cómo distintos tipos de organización gestionan su presencia en plataformas sociales. XX International Conference on Knowledge, Culture, and Change in Organizations.

Matosas-López, L., Luzardo-Briceño, M., Aguilar-Jiménez, A.-S., y Jaimes-Carrillo, L. (2021). Relaciones entre redes sociales y recursos digitales de instrucción en la universidad: comparativa España - Colombia. Pixel-Bit. Revista de Medios y Educación, 60(1), 77-93. https://doi.org/10.12795/pixelbit.77522

Matosas-López, L., y Romero-Ania, A. (2020). The Efficiency of Social Network Services Management in Organizations. An In-Depth Analysis Applying Machine Learning Algorithms and Multiple Linear Regressions. Applied Sciences, 10, 5167. https://doi.org/10.3390/app10155167

Matosas López, L. (2018). Variables of twitter's brand activity that influence audience spreading behavior of branded content. Esic Market Economics and Business Journal, 44(3), 525-546. https:// doi.org/10.7200/esicm.161.0491

McAndrew, F. T., y Jeong, H. S. (2012). Who does what on Facebook? Age, sex, and relationship status as predictors of Facebook use. Computers in Human Behavior, 28(6), 2359-2365. https:/ / doi.org/10.1016/J.CHB.2012.07.007

McBride, D. L. (2011). Risks and benefits of social media for children and adolescents. Journal of Pediatric Nursing: Nursing Care of Children and Families, 26(5), 498-499. https://doi.org/10.1016/J.PEDN.2011.05.001

McLuhan, M., y Nevitt, B. (1972). Take today; the executive as dropout. Harcourt Brace Jovanovich.

Mukherjee, K., y Banerjee, N. (2019). Social networking sites and customers' attitude towards advertisements. Journal of Research in Interactive Marketing, 13(4), 477-491. https://doi.org/10.1108/JRIM-06-2018-0081

Puertas Hidalgo, R., y Carpio Jiménez, L. (2016). Gestión de redes sociales por parte de las universidades categoría a en Ecuador. Iberian Conference on Information Systems and Technologies, CISTI, 2016-July. https://doi.org/10.1109/CISTI.2016.7521587 
Matosas-López, L. y Cuevas-Molano, E.

Propuestas para unas estrategias de marketing en redes sociales, más eficientes. El análisis de las cuentas corporativas universitarias

Quintana Pujalte, L., Sosa Valcarcel, A., y Castillo Esparcia, A. (2018). Acciones y estrategias de comunicación en plataformas digitales. El caso Cifuentes. Prisma Social, 22(3), 247-270. https://revistaprismasocial.es/article/view/2585

Raacke, J., y Bonds-Raacke, J. (2008). MySpace and Facebook: Applying the Uses and Gratifications Theory to Exploring Friend-Networking Sites. CyberPsychology y Behavior, 11(2), 169-174. https://doi.org/10.1089/cpb.2007.0056

Rubin, A. M. (1994). Media uses and effects: A uses-and-gratifications perspective. In B. J. Z. Dolf (Ed.), Media effects: Advances in theory and research (LEA's comm, pp. 417-436). Lawrence Erlbaum Associates Inc.

Ruggiero, T. E. (2000). Uses and Gratifications Theory in the 21st Century. Mass Communication and Society, 3(1), 3-37. https://doi.org/10.1207/S15327825MCS0301_02

Sánchez Carrero, J., y Contreras Pulido, P. (2012). De cara al prosumidor: producción y consumo empoderando a la ciudadanía 3.0. ICONO14, 10(3), 62-84. https://doi.org/10.7195/ri14.v10i3.210

Sandoval Romero, Y., y Aguaded Gómez, J. I. (2012). Nuevas audiencias, nuevas responsabilidades. La competencia mediática en la era de la convergencia digital. ICONO14, 10(3), 8-22. https://doi.org/10.7195/ri14.v10i3.197

Smock, A. D., Ellison, N. B., Lampe, C., y Wohn, D. Y. (2011). Facebook as a toolkit: A uses and gratification approach to unbundling feature use. Computers in Human Behavior, 27(6), 2322-2329. https:// doi.org/10.1016/J.CHB.2011.07.011

Tafesse, W. (2015). Content strategies and audience response on Facebook brand pages. Marketing Intelligence and Planning, 33(6), 927-943. https://doi.org/10.1108/MIP-07-2014-0135

Túñez López, M., Valdiviezo Abad, C., y Martínez Solana, Y. (2015). Las redes sociales en la gestión de la comunicación universitaria. Opción, 6, 852-874. https://dialnet.unirioja.es/servlet/articulo?codigo $=5758749$

Valerio Ureña, G., Herrera-Murillo, D. J., y Rodríguez-Martínez, M. D. C. (2014). Asociación entre el momento de publicación en las redes sociales y el engagement: Estudio de las universidades Mexicanas. Palabra Clave, 17(3), 749-772. https://doi.org/10.5294/pacla.2014.17.3.8

Wu, J., Chen, J., Chen, H., Dou, W., y Shao, D. (2019). What to say on social media and how: Effects of communication style and function on online customer engagement in China. Journal of Service Theory and Practice, 29(5-6), 691-707. https://doi.org/10.1108/JSTP-11-2018-0243 
Matosas-López, L. y Cuevas-Molano, E.

Propuestas para unas estrategias de marketing en redes sociales, más eficientes. El análisis de las cuentas corporativas universitarias

\section{AUTORES:}

\section{Luis Matosas-López:}

Profesor a tiempo completo en la Universidad Rey Juan Carlos (Acreditado por la ANECA como Profesor Contratado Doctor). Doctor en Economía, Master en Marketing, Licenciado en Investigación y Técnicas de Mercado y Diplomado en Ciencias Empresariales. Luis Matosas López ha realizado más de 20 publicaciones de relevancia, todas ellas relacionadas con el uso de las TICs y sus implicaciones en el plano de la gestión en organizaciones de distinto tipo. Entre estas destacan artículos en revistas indexadas en JCR y SJR (Q1 a Q3) así como capítulos de libro que han visto la luz en editoriales ubicadas en primeras posiciones de SPI como Tirant Lo Blanch, Pirámide, McGraw Hill, Dykinson, o Tecnos.

ORCID: https:/ / orcid.org/orcid-search/ search?searchQuery=0000-0001-7313-0146

Google Académico:

https://scholar.google.com/citations?hl=es\&user=AUBs43oAAAAJ

\section{Elena Cuevas-Molano:}

Doctora en Comunicación. Profesora de la Universidad Rey Juan Carlos desde el 2006. Imparte docencia dentro del departamento de Ciencias de la Comunicación y Sociología en los grados de Publicidad y RR.PP., Diseño Integral y Gestión de la Imagen; Protocolo, Organización de Eventos y Comunicación Corporativa. Ha desempeñado perfiles de responsable en departamentos de Publicidad, Imagen de Marca, y Marketing dentro de los sectores de medios de comunicación, telecomunicaciones y turismo, en entornos nacionales e internacionales.

ORCID: https:/ / orcid.org/orcid-search/ search?searchQuery=0000-0002-9876-8207 Google Académico:

https://scholar.google.com/citations?hl=es\&user=nvPCL8QAAAAJ 